\title{
The space of sampled ancestor trees QGSA2016
}

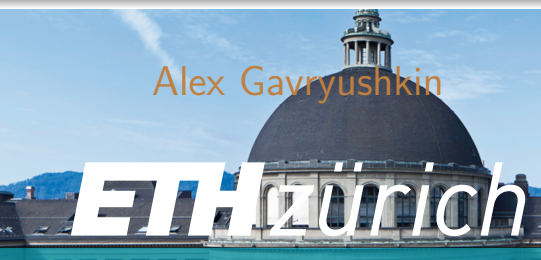

Joint work with

Alexei Drummond, the University of Auckland, NZ, Erick Matsen and Chris Whidden,

Fred Hutch Cancer Research Center, Seattle, WA, USA 


\section{Motivation}

- General statistics is at least 5 years ahead of phylostatistics. 


\section{Motivation}

- General statistics is at least 5 years ahead of phylostatistics.

- The discrete component of tree space is the bottleneck for tree search algorithms. 


\section{Motivation}

- General statistics is at least 5 years ahead of phylostatistics.

- The discrete component of tree space is the bottleneck for tree search algorithms.

- What's wrong with trees? 


\section{Motivation}

- General statistics is at least 5 years ahead of phylostatistics.

- The discrete component of tree space is the bottleneck for tree search algorithms.

- What's wrong with trees?

Same as above but with a mortarboard on

- MCMC algorithms

- Improving efficiency = smart proposals

- Point estimates AKA posterior summary

- Tree search methods in general

- Semi-convergence

- Valleys

- Terraces 


\section{Sampled ancestor tree}

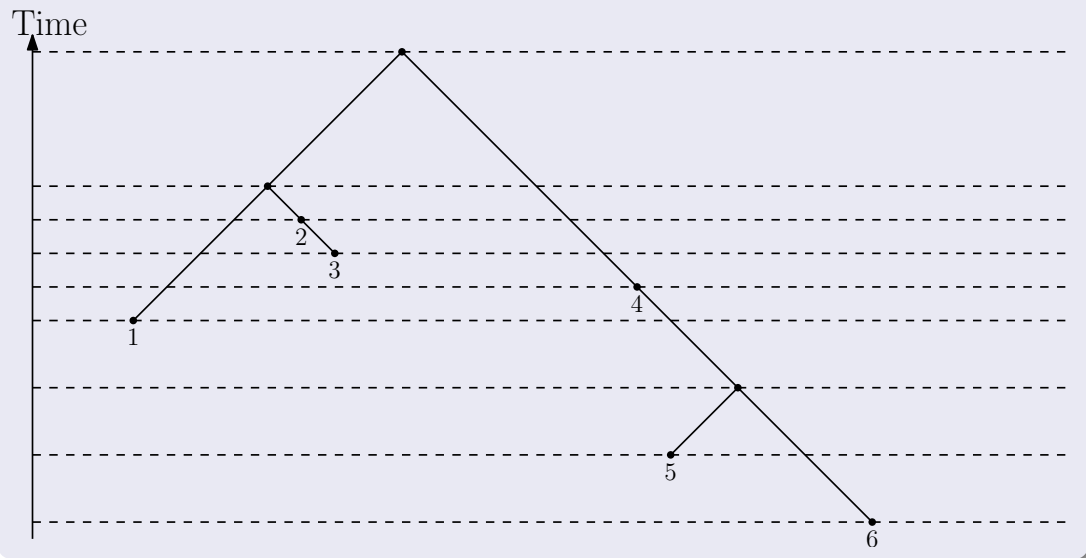




\section{Sampled ancestor tree graph}

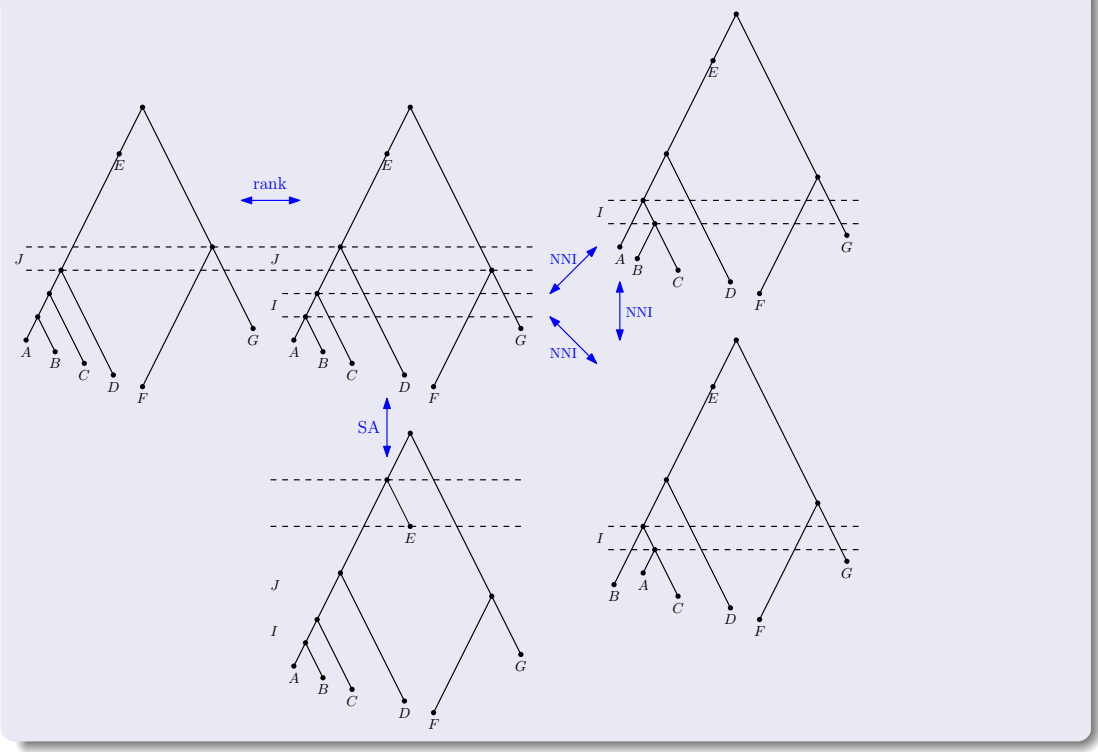




\section{Graph $=$ Metric space}




\section{Graph $=$ Metric space}

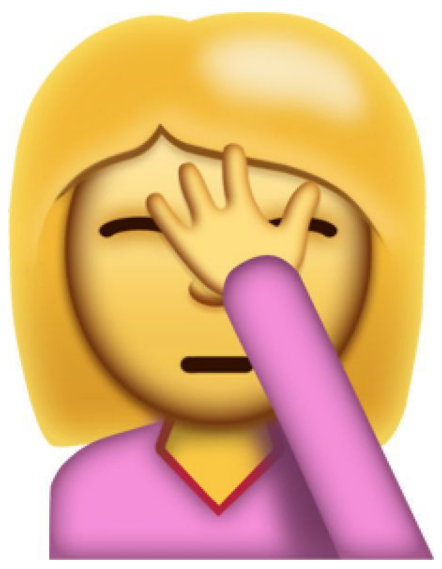




\section{What's wrong with the tree space?}

Answer

- Over 25 years to solve the complexity problem! 


\section{What's wrong with the tree space?}

Answer

- Over 25 years to solve the complexity problem!

- Over 7 erroneous "solutions" published on the way! 


\section{What's wrong with the tree space?}

Answer

- Over 25 years to solve the complexity problem!

- Over 7 erroneous "solutions" published on the way!

I'm talking about the NNI graph here. 


\section{What is actually "wrong"}

\section{The Split Theorem}

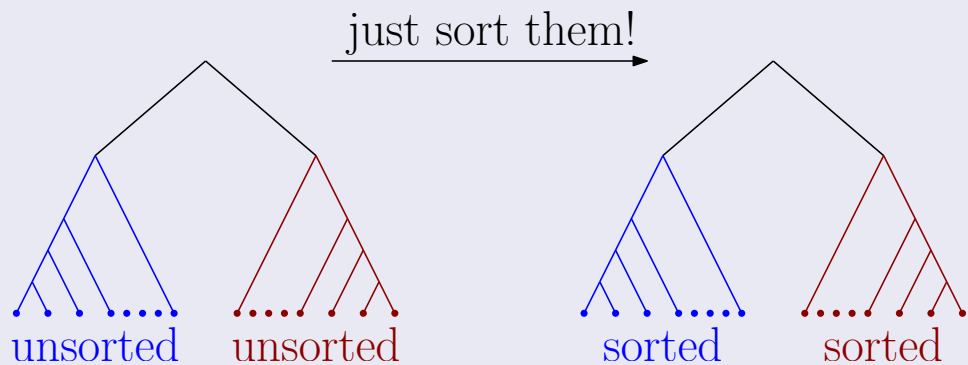




\section{What is actually "wrong"}

\section{Merge and sort trick}

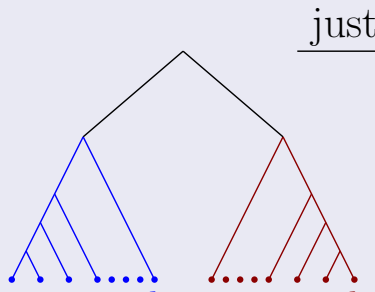

unsorted unsorted

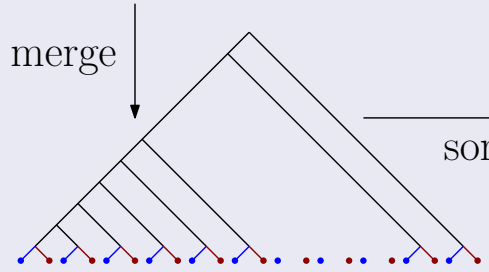

unsorted 


\section{Good news!}

Sampled ancestor trees (the SANNI graph) free from all these (G, Whidden, Matsen. bioRxiv, 2016)

- Split Theorem. Tick.

- Merge and sort trick. Tick. 


\section{Good news!}

Sampled ancestor trees (the SANNI graph) free from all these (G, Whidden, Matsen. bioRxiv, 2016)

- Split Theorem. Tick.

- Merge and sort trick. Tick.

\section{Even more good news}

Efficient approximate algorithm for computing shortest SANNI-paths. 


\section{What about branch lengths?}

\section{$\mathrm{G}$ and Drummond. JTB, 2016}

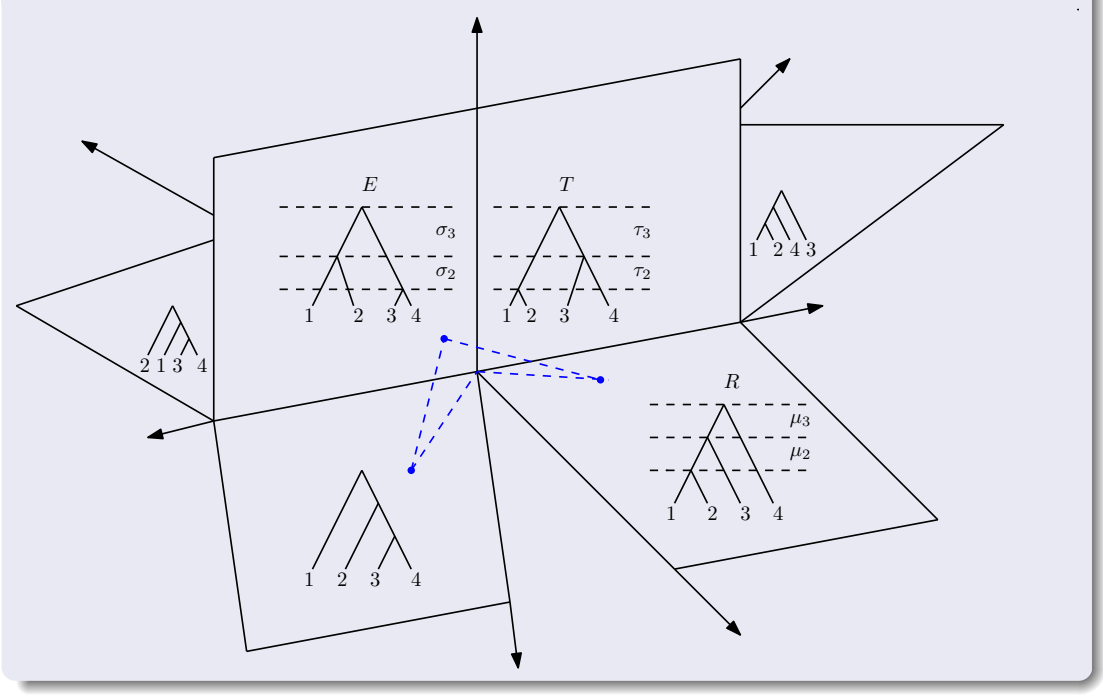




\section{Looks like a problem}

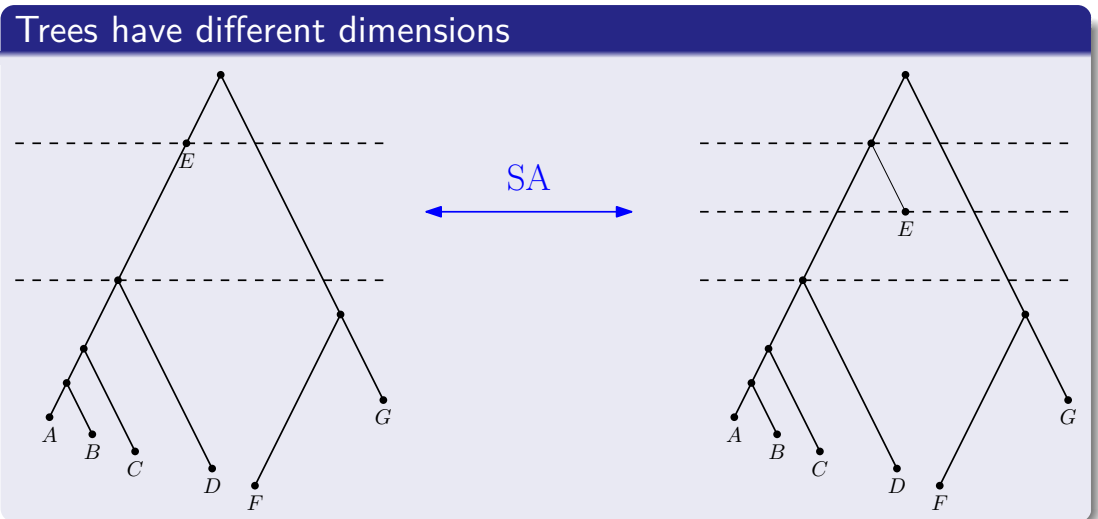




\section{Branch lengths are fine too!}

Stadler $(J T B, 2010)$ is cheating* anyway...

... so we can too: introduce "imaginary" nodes:
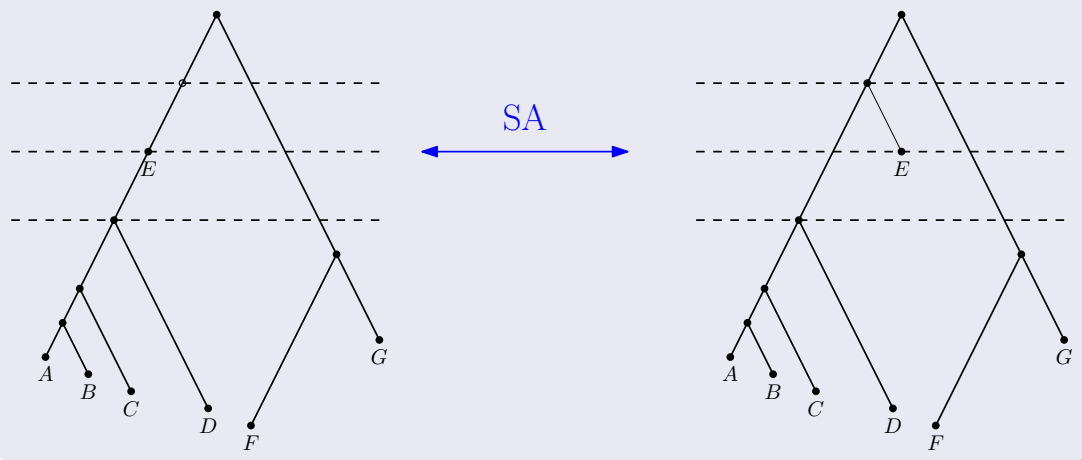


\section{Branch lengths are fine too!}

Stadler (JTB, 2010) is cheating* anyway...

... so we can too: introduce "imaginary" nodes:
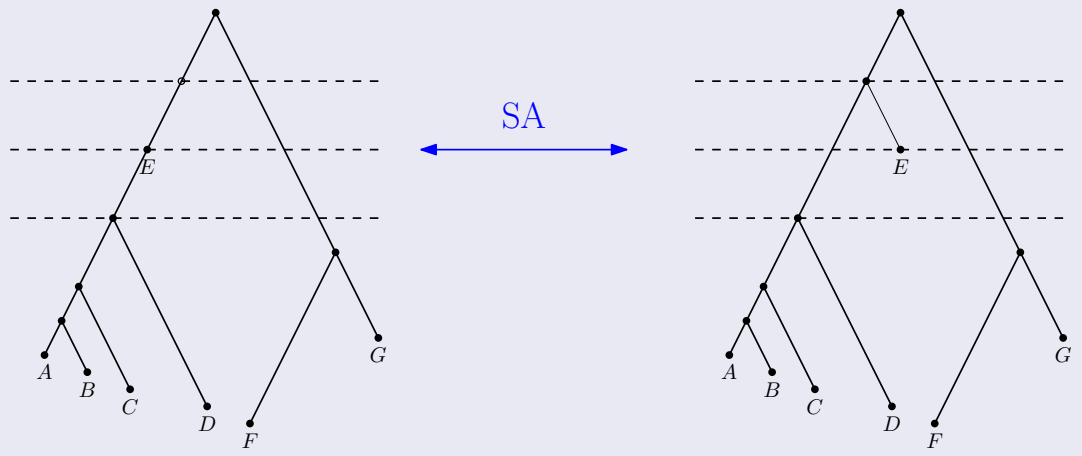

*by putting non-zero probability mass onto facets of the space 
- Introduced the SANNI graph on ranked sampled ancestor trees (to the best of our knowledge)

- Sampled ancestor trees and classical phylogenetic trees have different geometric and algorithmic properties

- Often, geometric and algorithmic results for classical trees do not scale to sampled ancestor trees

- Natural and efficient data structures

- Connections to other areas of math 
- Introduced the SANNI graph on ranked sampled ancestor trees (to the best of our knowledge)

- Sampled ancestor trees and classical phylogenetic trees have different geometric and algorithmic properties

- Often, geometric and algorithmic results for classical trees do not scale to sampled ancestor trees

- Natural and efficient data structures

- Connections to other areas of math

- Failed to prove that SANNI is NP-hard 


\section{References}

Li, Tromp, and Zhang

Some Notes on the Nearest Neighbour Interchange Distance.

Computing and Combinatorics, 343-351, 1996.

Dasgupta, He, Jiang, Li, Tromp, and Zhang

On Computing the Nearest Neighbor Interchange Distance

Discrete Mathematical Problems with Medical Applications, Vol. 55, 2000.

Alex Gavryushkin and Alexei Drummond

The space of ultrametric phylogenetic trees

Journal of Theoretical Biology, Vol. 402, 197-208, 2016

言四

Alex Gavryushkin, Chris Whidden, and Frederick A. Matsen IV

Combinatorics of discrete time-trees: algorithmic insights and open problems

bioRxiv, $2016 \quad \leftarrow \quad$ available as a blog post by Matsen

https://github.com/gavruskin/tauGeodesic

言 https://github.com/gavruskin/tTauCurvature 


\section{Thank you for your attention!}

\section{Funding}

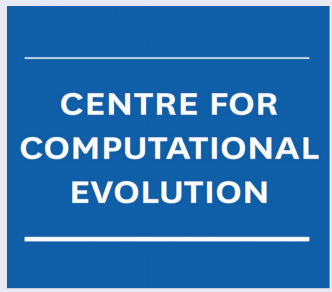

THE UNIVERSITY OF AUCKLAND

NEW ZEALAND 